\title{
Rastreo de alcoholismo en atención primaria
}

\author{
Screening for alcohol use disorders in primary care.
}

Coulton S. et al. BMJ 2006;332:511-517.

\section{Objetivo}

Evaluar la eficacia y los costos relativos de distintos métodos de rastreo de trastornos relacionados al consumo de alcohol.

\section{Lugar}

Seis hospitales generales del sur de Gales, Reino Unido.

\section{Diseño}

Estudio de corte transversal.

\section{Pacientes}

Hombres mayores de 18 años.

\section{Evaluación}

Para participar debían completar el cuestionario AUDIT (Alcohol Use Disorders Identification Tests) que aporta información sobre la frecuencia de consumo, problemas relacionados con el alcohol y síntomas de dependencia; y ser sometidos a pruebas bioquímicas. Se evaluó la sensibilidad, la especificidad y los valores predictivos positivos y negativos* (VPP, VPN) del AUDIT, así como los del dosaje de marcadores bioquímicos como la gamaglutamil-transpeptidasa (GGT) la aspartatoaminotransferasa (ASAT), el porcentaje de déficit de transferrina y el volumen corpuscular medio (VCM) en la detección de consumo peligroso, consumo compulsivo semanal y mensual definidos por un diario de consumo de alcohol en los últimos 180 días, el "time line follow back method" (TLFBM)- y dependencia al alcohol -definido por los criterios del Manual de Diagnóstico y Estadísticas de Salud Mental (DSM-IV). El TLFBM y el DSM-IV fueron considerados los tests de referencia.

\section{Resultados}

Los autores encontraron una correlación significativa (índice de Pearson* $r=0,74$ ) entre el consumo de alcohol y un resultado del AUDIT mayor a ocho puntos. La correlación con el dosaje de GGT $(r=0,20)$ y el porcentaje de déficit de transferrina $(r=$ 0,36 ) fue débil, no habiéndose hallado correlación significativa con el resto de los marcadores evaluados.

Para todas las categorías de problemas relacionados con el alcohol (consumo peligroso, consumo compulsivo y dependencia) el AUDIT mostró una sensibilidad, una especificidad y un VPP mayores que los marcadores bioquímicos. Ver tabla 1.

Tabla 1: resultados de la evaluación del cuestionario AUDIT para el rastreo de alcoholismo en Atención Primaria.

\begin{tabular}{l|c|c|c|c|c}
\multicolumn{1}{c|}{ Categoría } & Prevalencia estimada & Sensibilidad & Especificidad & \multicolumn{2}{c}{ Valor predictivo } \\
\cline { 3 - 5 } Consumo peligroso & & & $98 \%$ & $95 \%$ \\
\hline Compulsivo semanal & $34 \%$ & $69 \%$ & $90 \%$ & $71 \%$ \\
\hline Compulsivo mensual & $24 \%$ & $75 \%$ & $97 \%$ & $91 \%$ \\
\hline Dependencia & $35 \%$ & $66 \%$ & $83 \%$ & $41 \%$ \\
\hline
\end{tabular}

Asumiendo prevalencias de consumo de alcohol similares a las de este estudio, puede observarse, que un resultado positivo en el AUDIT es un indicador fuerte de problemas relacionados al alcohol (VPP muy alto) mientras que un resultado negativo es un buen indicador de ausencia de dependencia de alcohol (VPN alto).

El costo global de la implementación de cuestionario AUDIT (fotocopias, tiempo de la enfermera, ocupación del lugar donde es administrado) fue de 1,70 libras por paciente, mientras que el valor de los marcadores bioquímicos (extracción y análisis de resultados) fue de 5,25 a 27,25.

\section{Conclusiones}

El cuestionario AUDIT (ver cuadro 1) es una herramienta costoefectiva para la detección de trastornos relacionados al consumo de alcohol en atención primaria.

Fuente de financiamiento: Oficina Galesa de Investigación y Desarrollo.

\section{Comentario}

El cuestionario AUDIT (ver cuadro 1) fue validado en España ${ }^{1}$, donde se reportó una sensibilidad de $80 \%$ y una especificidad de $90 \%$. No sólo permite identificar individuos con dependencia sino también a los denominados consumidores de riesgo. También existe el cuestionario $\mathrm{CAGE}^{2}$ (ver la versión camuflada propuesta por Códoba y Altisent ${ }^{3}$ en el cuadro 2) que consta de cuatro preguntas y es utilizado más ampliamente en nuestro medio. En su versión española y con un punto de corte de 2 , tiene una sensibilidad del 49 al $97 \%$ y una especificidad del 79 al $96 \%$ para el diagnóstico de alcoholismo ${ }^{4,5,6}$. Se recomienda que sea administrado en forma "camuflada" entre otras ocho pre- guntas, para minimizar la sensación de incomodidad en el entrevistado y aumentar su sensibilidad.

\section{Conclusiones de los comentadores}

Los profesionales que trabajamos en el área de la atención primaria tenemos una situación estratégica para hacer rastreo de problemas relacionados con el alcohol y para actuar en consecuencia, por ejemplo mediante intervenciones breves $^{7}$, en los consumidores de riesgo o problemáticos que todavía no han desarrollado dependencia.

\section{María Rezzónico y Sergio Terrasa [ Servicio de Medicina Familiar y Preventiva del Hospital Italiano de Buenos Aires. maria.rozzonico@ hos-} pitalitaliano.org.ar]

Recibido el 20/02/07 y aceptado el 18/11/07

Rezzonico M y Terrasa S. Estrategias para el rastro de alcoholismo. Evid. actual. práct. ambul; 10(6): 172- 173, Nov-dic.2007. Comentado de: Coulton S. et all. Opportunistic screening for alcohol use disorders in primary care: comparative study. BMJ 2006;332:511-517. PMID: 16488896. Disponible en URL: http://www.bmj.com/cgi/reprint_abr/332/7540/511. Ultimo acceso: 09/12/07.

\section{Referencia}

1. Rubio G, Bermejo J, Caballero MC, Santo-Domingo J. Validación de la prueba para la identificación de trastornos por uso de alcohol (AUDIT) en atención primaria. Rev Clin Esp. 1998;198:11-4

2. Ewing JA. Detecting alcoholism. The CAGE questionnaire. JAMA 1984; 252:1905-7.

3. Citado por: Grupo de Educación Sanitaria y Promoción de la Salud del PAPPS. Guía de referencia rápida para abordar el abuso de alcohol. 2005. Sociedad Española de Medicina Familiar y Comunitaria. Disponible en URL: http://www.papps.org/publicaciones/abuso_alcohol.pdf (último acceso 08/12/07).

4. Campodarbe $F$ y col. Psicometría de la ansiedad, la depresión y el alcoholismo en Atención Primaria. Semergen 25 (3): $209-225$.

5. Rodríguez-Martos A, Navarro RM, Vecino C, Pérez R. Validación de los cuestionarios KFA (CBA) y CAGE para el diagnóstico del alcoholismo. Drogalcohol 1986; 11: 132-9 
Cuadro 1: cuestionario AUDIT validado en España. Se marca con un círculo la respuesta más correcta en cada caso.

Ahora voy a hacerle algunas preguntas sobre el consumo de bebidas alcohólicas. Me refiero a bebidas como la sidra, la cerveza, la cerveza llamada sin alcohol, vino, licores, coñac, ginebra y otros. Para entendernos, a partir de ahora, cuando nos refiramos a una bebida alcohólica será el equivalente a una caña de cerveza, o una copa de vino, un vermout, una copa de licor de frutas, medio cubalibre o medio whisky.

\section{Marque con un círculo la respuesta más correcta en su caso:}

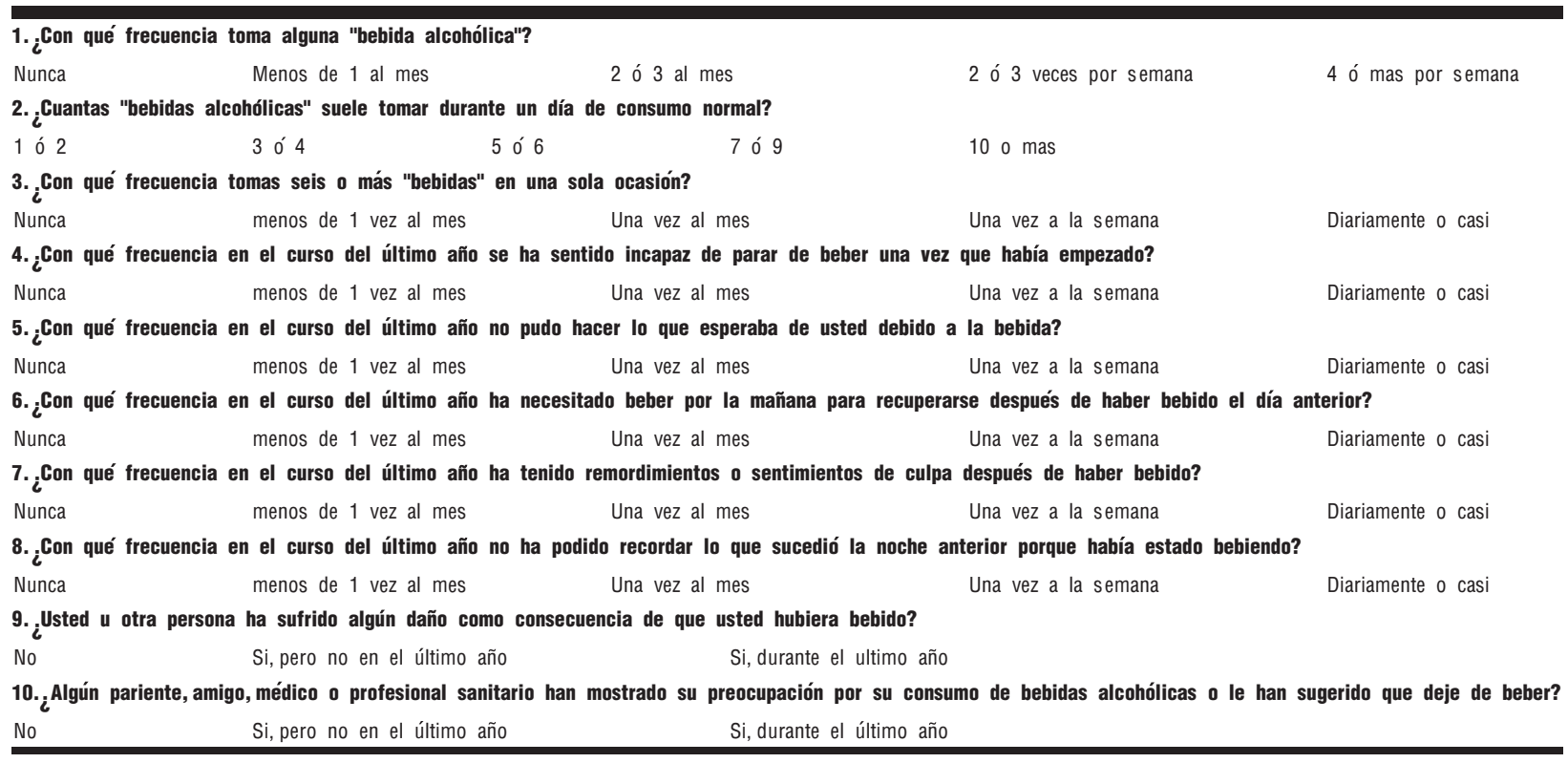

Los resultados del cuestionario se analizan asignando una puntuación de 0, 1, 2, 3 ó 4 a las ocho primeras preguntas y 0, 2 ó 4 puntos a las dos últimas. Valores entre 8 y 20 puntos sugieren un consumo perjudicial, siendo indicativos de dependencia los superiores a 20. Las preguntas 2 y 3 permiten valorar consumos de riesgo que por la cantidad ingerida puedan poner en peligro al bebedor o a terceros, independientemente de la presencia de dependencia y aún con valores totales del cuestionario menores a ocho puntos.

Fuente: Centeno A y col. El médico de Atención Primaria en la prevención y tratamiento de los problemas relacionados con el consumo de alcohol. Medicina General 2001; 32: 233-240.

Cuadro 2: se describe en negritas la versión validada en España del cuestionario CAGE. Una de las alternativas para administrarlo es hacerlo en forma camuflada entre otras preguntas como las que se describen a continuación.

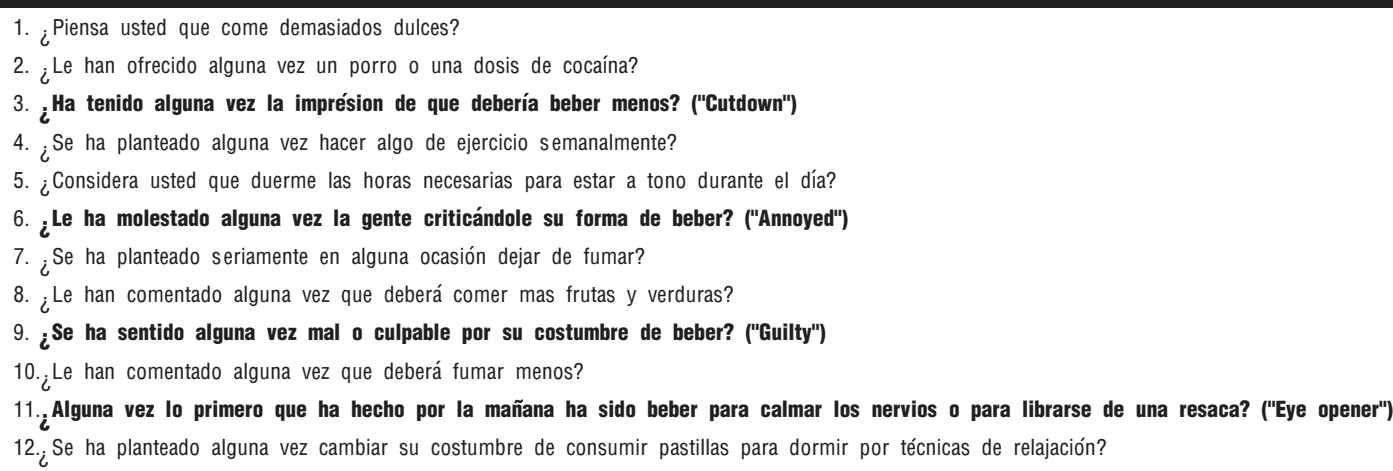

La respuesta positiva a dos de las cuatro preguntas tiene una sensibilidad del 49 al $97 \%$ y una especificidad del 79 al $96 \%$ para el diagnostico de alcoholismo.

Fuente: Campodarbe F y col. Psicometría de la ansiedad, la depresión y el alcoholismo en Atención Primaria. Semergen 25 (3): $209-225$. Grupo de Educación Sanitaria y Promoción de la Salud del PAPPS. Guía de referencia rápida para abordar el abuso de alcohol. 2005. Sociedad Española de Medicina Familiar y Comunitaria.

6. Centeno A y col. El médico de Atención Primaria en la prevención y tratamiento de los problemas relacionados con el consumo de alcohol. Medicina General 2001; 32: 233240. Disponible en URL: http://www.medicinageneral.org/marzo2001/233-240.pdf (último acceso 08/12/07).

7. Grupo de Educación Sanitaria y Promoción de la Salud del PAPPS. Guía de referencia rápida para abordar el abuso de alcohol. 2005. Sociedad Española de Medicina Familiar y Comunitaria. Disponible en URL: http://www.papps.org/publicaciones/abuso_alcohol.pdf (último acceso 08/12/07). 